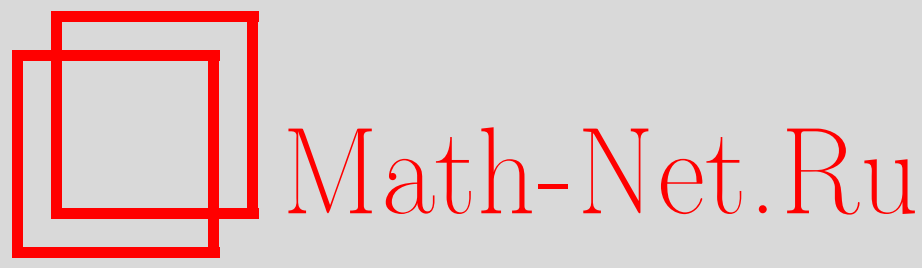

И. В. Стасюк, О. Б. Гера, Асимметричная модель Хаббарда в методе производящего функционала: спектральные функции в пределе Фаликова-Кимбала, ТМФ, 2008, том 154, номер 1, 164-182

DOI: https://doi.org/10.4213/tmf6158

Использование Общероссийского математического портала Math-Net.Ru подразумевает, что вы прочитали и согласны с пользовательским соглашением http://www . mathnet.ru/rus/agreement

Параметры загрузки:

IP : 18.207 .199 .55

26 апреля 2023 г., 15:10:09

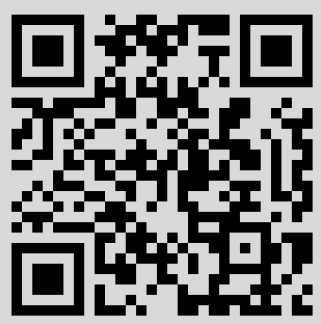




\section{АСИММЕТРИЧНАЯ МОДЕЛЬ ХАББАРДА В МЕТОДЕ ПРОИЗВОДЯЩЕГО ФУНКЦИОНАЛА: СПЕКТРАЛЬНЫЕ ФУНКЦИИ В ПРЕДЕЛЕ ФАЛИКОВА-КИМБАЛА}

В рамках теории динамического среднего поля исследованы плотности состояний фермионной и бозонной ветвей спектра асимметричной модели Хаббарда, которая применяется для описания сильно коррелированной двухсортной $(A, B)$-системы ферми-частиц (электронов). Для решения эффективной одноузельной задачи развит приближенный аналитический подход, который базируется на методе производящего функционала Каданова-Бейма. Данная методика позволяет построить неприводимую часть (массовый оператор) функции Грина частиц в виде формального разложения по степеням когерентного потенциала. В первом порядке схема воспроизводит так называемое обобщенное приближение Хаббард-ІІІ. С целью ее улучшения развит самосогласованный способ расчета как фермионной, так и бозонной функций Грина. При $U \rightarrow \infty$ в пределе Фаликова-Кимбала асимметричной модели Хаббарда, когда частицы сорта $B$ становятся локализованными, найдены спектральные плотности состояний $\rho_{B}$ и $\rho_{A B}$ обеих ветвей, рассмотрено изменение их формы в зависимости от температуры и концентраций частиц. Путем сравнения с точными термодинамическими зависимостями $\mu_{B}\left(n_{B}\right)$ установлены пределы применимости самосогласованного обобщенного приближения Хаббард-III.

Ключевые слова: сильно коррелированные системы, асимметричная модель Хаббарда, динамическое среднее поле, производящий функционал, спектральные функции.

\section{1. ВВЕДЕНИЕ}

Решеточные модели с хаббардовскими короткодействующими взаимодействиями используются для исследования физических свойств сильно коррелированных электронных систем; однозонную модель Хаббарда и бесспиновую модель ФаликоваКимбала $(\Phi \mathrm{K})$ можно рассматривать как простейшие модели такого типа (см., например, [1]-[3]). В настоящей работе эти модели объединены в единую асимметричную модель Хаббарда (AMX) с разными параметрами переноса и разными значениями химических потенциалов, которая описывает систему с двумя сортами подвижных частиц (электронов, ионов и т.п.). Данная модель может применяться

* Институт физики конденсированных систем НАН Украины, Львов, Украина. E-mail: ista@icmp.lviv.ua, hera@icmp.lviv.ua 
к системам со смешанной валентностью [4], а также к ионным проводникам. Гамильтониан модели имеет следующий вид:

$$
\widehat{H}=\sum_{i} \widehat{H}_{i}+\sum_{i j \sigma} t_{i j}^{\sigma} a_{i \sigma}^{\dagger} a_{j \sigma},
$$

где

$$
\widehat{H}_{i}=U n_{i \uparrow} n_{i \downarrow}-\sum_{\sigma \in\{\downarrow, \uparrow\}} \mu_{\sigma} n_{i \sigma} .
$$

Здесь электронный перенос (перенос частиц) описывается на языке фермионных операторов рождения и уничтожения $a_{i \sigma}^{\dagger}, a_{j \sigma}$ и характеризуется параметром $t_{i j}^{\sigma}$, зависящим от спинового индекса $\sigma$. Одноузельная часть оператора $\widehat{H}_{i}$ содержит локальное отталкивание $U$ и энергию электронов в локализованных состояниях, включенную в химические потенциалы $\mu_{\sigma}\left(n_{i \sigma}=a_{i \sigma}^{\dagger} a_{i \sigma}\right)$. При использовании модели для описания систем с двумя сортами ферми-квазичастиц спиновые индексы $\sigma=\uparrow, \downarrow$ заменяются индексами сорта частиц $\sigma=A, B$. Основное отличие АМХ от обычной модели Хаббарда состоит в том, что интегралы переноса и химические потенциалы зависят от сорта частиц $\left(t_{i j}^{A} \neq t_{i j}^{B}, \mu_{A} \neq \mu_{B}\right)$. При предельном переходе $t_{i j}^{B} \rightarrow 0$, когда частицы сорта $B$ становятся неподвижными, модель (1) переходит в $Ф$ К-модель. При этом, если модель применяется к электронам в различных орбитальных состояниях, часто вводят единый химический потенциал, положив $\mu_{A}=\mu-\varepsilon_{A} ; \mu_{B}=\mu-\varepsilon_{B}[3]$.

$\mathrm{K}$ настоящему времени исследованы только некоторые частные случаи АМХ. Можно отметить анализ основного состояния модели с точки зрения неустойчивости однородной фазы [5]. Рассматривался также случай сильного одноузельного отталкивания $U$, в рамках которого можно свести гамильтониан исходной модели к эффективному гамильтониану анизотропной модели Гейзенберга с антиферромагнитным взаимодействием [4], [6], [7]. Некоторые термодинамические свойства модели исследовались в одномерном случае [7], [8], а в пределе бесконечной размерности пространства $(d \rightarrow \infty)$ проводились расчеты одночастичной плотности состояний с использованием метода уравнений движения для функций Грина [9], рассматривались также критерии перехода металл-диэлектрик [10].

В настоящей работе мы продолжим исследование AMX при $d \rightarrow \infty$ в рамках метода динамического среднего поля (который является точным в этом пределе). В этом случае полная неприводимая часть $\Xi_{\sigma}\left(\omega_{n}\right)$ одночастичной функции Грина не зависит от волнового вектора, и ее нахождение для кристаллической решетки сводится к одноузельной задаче [11]. Решение этой задачи дополняется соотношением

$$
G_{\sigma}\left(\omega_{n}\right)=\frac{1}{\Xi_{\sigma}^{-1}\left(\omega_{n}\right)-J_{\sigma}\left(\omega_{n}\right)},
$$

вытекающим из уравнения Ларкина (здесь $G_{\sigma}\left(\omega_{n}\right)$ - одноузельная функция Грина, $J_{\sigma}\left(\omega_{n}\right)$ - когерентный потенциал), а также условием совпадения решеточной $G_{i i}^{\sigma}$ и одноузельной функций Грина:

$$
G_{\sigma}\left(\omega_{n}\right)=\int_{-\infty}^{+\infty} \frac{d t \rho_{\sigma}^{0}(t)}{\Xi_{\sigma}^{-1}\left(\omega_{n}\right)-t}
$$


где $\rho_{\sigma}^{0}(t)$ - невозмущенная плотность зонных состояний.

Известен ряд численных методов решения одноузельной задачи (например, квантовый метод Монте-Карло [12]-[14], метод точной диагонализации [15], [16], метод численной ренормгруппы [17]). Однако эти подходы обладают рядом ограничений, и в связи с развитием в последнее время методик расчета электронной структуры на основе метода функционала плотности [18], [19] возникает необходимость разработки новых быстрых алгоритмов. Такими являются, в частности, аналитические аппроксимации для функций Грина [20]-[25].

Мы базируемся на работе [26], в которой для улучшения аналитического подхода развивалась методика производящего функционала в применении к одноузельной задаче. Основу этой методики составляет схема Каданова-Бейма [27], развитая и переформулированная Изюмовым и Чащиным [28]-[31] в применении к решеточным системам с сильными корреляциями (описываемым, в частности, моделями Гейзенберга и Хаббарда). Данная методика позволяет получать замкнутое уравнение в функциональных производных для неприводимой части функции Грина. Его решение строится итерационным методом в виде разложения по степеням когерентного потенциала $J_{\sigma}$. Как показано в [26], уже первая итерация приводит после некоторых упрощений к так называемому обобщенному приближению Хаббард-III (GH3), которое было получено ранее с помощью процедуры разновременных расцеплений неприводимых функций Грина в методе уравнений движения [9], [32] (при половинном заполнении и в симметричном случае GH3-приближение сводится к обычному приближению Хаббард-III).

Выход за пределы GH3-приближения в рамках используемого подхода может быть осуществлен путем более строгого расчета одноузельной функции Грина, построенной на бозонных операторах Хаббарда. Следуя идее, изложенной в [31], в настоящей работе уравнения для такой функции мы получаем путем функционального дифференцирования средних от бозонных $X$-операторов по фиктивным полям. Связанная с этим неопределенность аналогична тому или иному выбору приоритетов спариваний в стандартной диаграммной технике, что приводит к различному виду получаемых уравнений. Для решения этой проблемы проанализированы результаты, которые соответствуют различным исходным средним; использована также процедура симметризации [31]. Бозонные функции Грина определяются приближенно с точностью до первого порядка по когерентному потенциалу.

Определение бозонной и фермионной функций Грина на основе полученной системы уравнений позволяет улучшить GH3-приближение, делая его самосогласованным. С целью обоснования и апробации развиваемого подхода в настоящей работе проводится расчет зависимостей химического потенциала $\mu_{B}$ от концентрации $n_{B}$ локализованных частиц АMX в $\Phi$ К-пределе $\left(t_{B}=0\right)$ при разных температурах и различных значениях химического потенциала $\mu_{A}$. Полученные кривые сравниваются с результатами, вытекающими из точного выражения для термодинамического потенциала в этом пределе [32], а также с зависимостями, найденными в других приближениях, включая обычное GH3-приближение [9]. В той области значений параметров, где самосогласованное GH3-приближение является удовлетворитель- 
ным, рассчитаны бозонные и фермионные спектральные функции (плотности состояний $\rho_{A B}$ и $\rho_{B}$ ), проанализированы зависимости спектра возбуждений от концентраций частиц и температуры.

\section{2. ЭФФЕКТИВНАЯ ОДНОУЗЕЛЬНАЯ ЗАДАЧА В РАМКАХ ПОДХОДА ПРОИЗВОДЯЩЕГО ФУНКЦИОНАЛА}

Одноузельная задача для определения электронной функции Грина в методе динамического среднего поля формулируется обычно на языке эффективного статистического оператора

$$
\mathrm{e}^{-\beta \widehat{H}_{\text {eff }}}=\mathrm{e}^{-\beta \widehat{H}_{0}} \mathcal{T}_{\tau} \exp \left[-\int_{0}^{\beta} d \tau \int_{0}^{\beta} d \tau^{\prime} \sum_{\sigma} J_{\sigma}\left(\tau-\tau^{\prime}\right) a_{\sigma}^{\dagger}(\tau) a_{\sigma}\left(\tau^{\prime}\right)\right] \equiv \mathrm{e}^{-\beta \widehat{H}_{0}} \hat{\sigma}(\beta)
$$

где $H_{0}$ - одноузельная часть гамильтониана $(1), J_{\sigma}\left(\tau-\tau^{\prime}\right)$ - когерентный потенциал. Целью расчета функции Грина

$$
G_{\sigma}=\left\langle\mathcal{T}_{\tau} a_{\sigma}^{\dagger}(\tau) a_{\sigma}\left(\tau^{\prime}\right)\right\rangle
$$

является установление функциональной связи между функцией $G_{\sigma}$ и потенциалом $J_{\sigma}$, которая вместе с (3) и (4) позволяет получить замкнутую систему уравнений.

Воспользуемся представлением операторов рождения и уничтожения фермионов через операторы Хаббарда:

$$
a_{\sigma}=X^{0 \sigma}+\zeta X^{\bar{\sigma} 2}, \quad a_{\sigma}^{\dagger}=X^{\sigma 0}+\zeta X^{2 \bar{\sigma}}
$$

здесь индексы и знаки определены следующим образом: $\bar{\sigma}=B, \zeta=+$ для $\sigma=A$; $\bar{\sigma}=A, \zeta=-$ для $\sigma=B$; индексы 0 и 2 обозначают соответственно незанятое и двукратно занятое состояние узла. Тогда

$$
H_{0}=U X^{22}-\sum_{\sigma} \mu_{\sigma}\left(X^{\sigma \sigma}+X^{22}\right)
$$

и функция Грина (8) приобретает вид суммы четырех слагаемых:

$$
\begin{aligned}
G_{\sigma}\left(\tau-\tau^{\prime}\right)=\left\langle T_{\tau} X^{\sigma 0}(\tau) X^{0 \sigma}\left(\tau^{\prime}\right)\right\rangle+\zeta\left\langle T_{\tau} X^{\sigma 0}(\tau) X^{\bar{\sigma} 2}\left(\tau^{\prime}\right)\right\rangle+ \\
+\left\langle T_{\tau} X^{2 \bar{\sigma}}(\tau) X^{\bar{\sigma} 2}\left(\tau^{\prime}\right)\right\rangle+\zeta\left\langle T_{\tau} X^{2 \bar{\sigma}}(\tau) X^{0 \sigma}\left(\tau^{\prime}\right)\right\rangle .
\end{aligned}
$$

Для расчета функции (9), построенной на $X$-операторах, можно применить температурную теорию возмущений, разлагая по степеням когерентного потенциала $J_{\sigma}$. Такой подход соответствует приближению сильной связи, которое для систем с хаббардовским взаимодействием использовалось в ряде работ (см., например, [33]). В методе производящего функционала данному разложению соответствует упомянутая выше итерационная процедура определения неприводимой по Ларкину части $\Xi_{\sigma}$ функции Грина [26], [28]-[31]. 
С целью упрощения дальнейшего изложения мы рассмотрим предельный случай $U \rightarrow \infty$, в рамках которого исключаются двукратно занятые состояния узлов, и задача сводится к определению функции Грина $G^{\sigma 0,0 \sigma} \equiv\left\langle\mathcal{T}_{\tau} X^{\sigma 0}(\tau) X^{0 \sigma}\left(\tau^{\prime}\right)\right\rangle$.

Введем зависящие от времени флуктуирующие поля $v_{\gamma}(\tau)$, сопряженные величинам, описываемым бозонными операторами $X^{\gamma}(\gamma=00, \sigma \sigma, \sigma \bar{\sigma})$. Статистическая сумма одноузельной задачи запишется при этом в виде

$$
Z \rightarrow Z_{V}=\operatorname{Sp}\left(\mathrm{e}^{-\beta H_{\text {eff }}} \mathcal{T}_{\tau} \mathrm{e}^{-V}\right)
$$

где

$$
V=\int_{0}^{\beta} d \tau V(\tau)=\int_{0}^{\beta} d \tau \sum_{\gamma} v_{\gamma}(\tau) X^{\gamma}(\tau),
$$

а вычисление средних от произведений $X$-операторов осуществляется согласно формуле

$$
\langle\ldots\rangle_{V}=\frac{\operatorname{Sp}\left(\mathcal{T}_{\tau} \mathrm{e}^{-\beta H_{\text {eff }}} \ldots \mathrm{e}^{-V}\right)}{\operatorname{Sp}\left(\mathcal{T}_{\tau} \mathrm{e}^{-\beta H_{\text {eff }}} \mathrm{e}^{-V}\right)} .
$$

Средние значения бозонных операторов $X^{\gamma}$ выражаются как функциональные производные по соответствующим полям $v_{\gamma}$ от производящего функционала $\Phi_{V}=$ $\ln Z_{V},\left\langle X^{\gamma}\right\rangle=-\delta \Phi_{V} /\left.\delta v_{\gamma}(\tau)\right|_{v \rightarrow 0}$. Чтобы получить средние для системы с исходным гамильтонианом, поля $v_{\gamma}$ полагаются после дифференцирования равными нулю.

Пользуясь выражениями для производных от функционала $\Phi_{V}$, легко получить соотношения, с помощью которых записывается произвольный коррелятор (функция Грина) высшего порядка, содержащий бозонный оператор Хаббарда:

$$
\left\langle\mathcal{T}_{\tau} X^{\alpha}\left(\tau_{1}\right) \ldots\right\rangle_{V}=-\frac{\delta\left\langle\mathcal{T}_{\tau} \ldots\right\rangle_{V}}{\delta v_{\alpha}\left(\tau_{1}\right)}+\left\langle\mathcal{T}_{\tau} \ldots\right\rangle_{V}\left\langle\mathcal{T}_{\tau} X^{\alpha}\left(\tau_{1}\right)\right\rangle_{V}
$$

При переходе к усреднению с гамильтонианом $H_{0}$,

$$
\left\langle\mathcal{T}_{\tau} \ldots\right\rangle_{V}=\frac{\left\langle\mathcal{T}_{\tau} \ldots \hat{\sigma}(\beta) \mathrm{e}^{-V}\right\rangle_{0}}{\left\langle\hat{\sigma}(\beta) \mathrm{e}^{-V}\right\rangle_{0}}
$$

и при разложении в ряд $\mathcal{T}_{\tau}$-экспоненты возникают, как обычно, средние от произведений $X$-операторов. Вычислим их, используя предложенную в [26] так называемую рекуррентную форму теоремы Вика. В соответствии с [26] результат спаривания произвольно взятого недиагонального оператора $X^{\sigma 0}(\tau)$ с остальными (при возвращении к усреднению с полным гамильтонианом) задается соотношением

$$
\begin{aligned}
& \left\langle\mathcal{T}_{\tau} X^{\sigma 0}(\tau) X^{\gamma_{1}}\left(\tau_{1}\right) \ldots X^{\gamma_{n}}\left(\tau_{n}\right)\right\rangle= \\
& =\sum_{i} g^{\sigma 0}\left(\tau-\tau_{i}\right)\left\langle\mathcal{T}_{\tau} X^{\gamma_{1}}\left(\tau_{1}\right) \ldots\left[X^{\sigma 0}, X^{\gamma_{i}}\right]_{\tau_{i}}^{ \pm} \ldots X^{\gamma_{n}}\left(\tau_{n}\right)\right\rangle(-1)^{p_{i}}+ \\
& \quad+\int_{0}^{\beta} d \tau^{\prime} \int_{0}^{\beta} d \tau^{\prime \prime} \sum_{\sigma_{1}} J_{\sigma_{1}}\left(\tau^{\prime}-\tau^{\prime \prime}\right)\left\langle\mathcal { T } _ { \tau } X ^ { \gamma _ { 1 } } ( \tau _ { 1 } ) \ldots X ^ { \gamma _ { n } } ( \tau _ { n } ) \left\{ g^{\sigma 0}\left(\tau-\tau^{\prime \prime}\right) \times\right.\right. \\
& \left.\left.\quad \times\left[X^{\sigma 0}, a_{\sigma_{1}}^{\dagger}\right]_{\tau^{\prime}}^{+} a_{\sigma_{1}}\left(\tau^{\prime \prime}\right)-g^{\sigma 0}\left(\tau-\tau^{\prime \prime}\right) a_{\sigma_{1}}^{\dagger}\left(\tau^{\prime}\right)\left[X^{\sigma 0}, a_{\sigma_{1}}\right]_{\tau^{\prime \prime}}^{+}\right\}\right\rangle+
\end{aligned}
$$




$$
+\int_{0}^{\beta} d \tau^{\prime} g^{\sigma 0}\left(\tau-\tau^{\prime}\right)\left\langle\mathcal{T}_{\tau} X^{\gamma_{1}}\left(\tau_{1}\right) \ldots X^{\gamma_{n}}\left(\tau_{n}\right)\left[X^{\sigma 0}, \widehat{V}\right]_{\tau^{\prime}}^{-}\right\rangle
$$

Здесь множитель $(-1)^{p_{i}}$ определяется числом ферми-перестановок $p_{i}$ оператора $X^{\sigma 0}(\tau)$ из исходного положения в положение непосредственно слева от оператора, с которым совершается спаривание; $g^{\sigma 0}(\tau)$ - невозмущенная функция Грина (в частотном представлении $\left.g^{\sigma 0}\left(\omega_{n}\right)=\left(i \omega_{n}+\mu_{\sigma}\right)^{-1}\right)$.

Соотношение (15) имеет смысл уравнения, связывающего средние от произведений $X$-операторов (в том числе функции Грина) с функциями высших порядков. Такого типа уравнения, характерные как для мацубаровских, так и для зубаревских двухвременных функций Грина (где они формируют известную, в частности, еще с работы Зубарева [34] бесконечную цепочку уравнений), получаются обычно в методе уравнений движения.

\section{3. ОДНОУЗЕЛЬНАЯ ФЕРМИОННАЯ ФУНКЦИЯ ГРИНА}

Применим соотношение $(15)$ к функции Грина $\left\langle\mathcal{T}_{\tau} X^{\sigma 0}(\tau) X^{0 \eta}\left(\tau^{\prime}\right)\right\rangle_{V}$ (здесь индексы $\sigma$ и $\eta$ могут принимать значения $A$ или $B$ ). Используем явные выражения для коммутаторов (антикоммутаторов) и примем во внимание, что $\left[X^{\sigma 0}, a_{\sigma_{1}}^{\dagger}\right]^{+} \rightarrow 0$ в отсутствии двукратно заполненных состояний (здесь $v_{0} \equiv v_{00}, v_{\sigma} \equiv v_{\sigma \sigma}, v \equiv v_{B A}$, $\left.\bar{v} \equiv v_{A B}\right)$. Выражая корреляторы $\left\langle\mathcal{T}_{\tau} X^{\sigma 0}\left(\tau_{1}\right)\left(X^{00}+X^{\sigma \sigma}\right)_{\tau_{2}} X^{0 \eta}\left(\tau^{\prime}\right)\right\rangle$ и $\left\langle\mathcal{T}_{\tau} X^{\bar{\sigma} 0}\left(\tau_{1}\right) \times\right.$ $\left.X^{\sigma \bar{\sigma}}\left(\tau_{2}\right) X^{0 \eta}\left(\tau^{\prime}\right)\right\rangle$ через исходную функцию Грина с помощью функциональных производных по полям $v_{\gamma}$, получим для нее уравнение (см. [26]), которое в матричном представлении имеет простой вид

$$
\hat{g}^{-1} \widehat{G}+\hat{A}_{0} \hat{J} \widehat{G}+\hat{A}^{\prime} \hat{J} \widehat{G}=-\hat{A}_{0} .
$$

Здесь

$$
\widehat{G}\left(\tau, \tau^{\prime}\right)=\left(\begin{array}{ll}
G_{V}^{B 0,0 B} & G_{V}^{B 0,0 A} \\
G_{V}^{A 0,0 B} & G_{V}^{A 0,0 A}
\end{array}\right), \quad \hat{J}\left(\tau, \tau^{\prime}\right)=\left(\begin{array}{cc}
J_{B}\left(\tau-\tau^{\prime}\right) & 0 \\
0 & J_{A}\left(\tau-\tau^{\prime}\right)
\end{array}\right)
$$

$G_{V}^{\sigma 0,0 \eta}\left(\tau, \tau^{\prime}\right) \equiv\left\langle\mathcal{T}_{\tau} X^{\sigma 0}(\tau) X^{0 \eta}\left(\tau^{\prime}\right)\right\rangle_{V}, \hat{A}^{\prime}-$ дифференциальный оператор,

$$
\hat{A}^{\prime}\left(\tau, \tau^{\prime}\right)=\delta\left(\tau-\tau^{\prime}\right)\left(\begin{array}{cc}
\frac{\delta}{\delta v_{0 B}(\tau)} & \frac{\delta}{\delta v(\tau)} \\
\frac{\delta}{\delta \bar{v}(\tau)} & \frac{\delta}{\delta v_{0 A}(\tau)}
\end{array}\right), \quad \frac{\delta}{\delta v_{0 \sigma}}=\frac{\delta}{\delta v_{0}}+\frac{\delta}{\delta v_{\sigma}}
$$

матрица $\hat{A}_{0}=\hat{A}^{\prime} \Phi_{V}$ является результатом действия оператора $\hat{A}^{\prime}$ на производящий функционал $\Phi_{V}$. Введена также матрица обратной невозмущенной функции Грина

$$
\begin{aligned}
\hat{g}^{-1}\left(\tau, \tau^{\prime}\right)= & \left(\begin{array}{cc}
\left(g^{B 0}\right)^{-1}\left(\tau-\tau^{\prime}\right) & 0 \\
0 & \left(g^{A 0}\right)^{-1}\left(\tau-\tau^{\prime}\right)
\end{array}\right)+ \\
& +\left(\begin{array}{cc}
v_{0}(\tau)-v_{B}(\tau) & -\bar{v}(\tau) \\
-v(\tau) & v_{0}(\tau)-v_{A}(\tau)
\end{array}\right) \delta\left(\tau-\tau^{\prime}\right) .
\end{aligned}
$$

Здесь (и ниже) произведение матриц содержит интегрирование по внутреннему временно́му аргументу. 
Перейдем по аналогии с функцией (3) от функции Грина $\widehat{G}$ к ее неприводимой по Ларкину части $\widehat{\Xi}$. Переписывая уравнение (16) в форме функционального дифференциального уравнения, где оператор $\hat{A}^{\prime}$ действует на обратную матричную функцию Грина $\widehat{G}^{-1}$, мы можем получить уравнение для обратной неприводимой части [26]

$$
\widehat{\Xi}^{-1}=\widehat{G}_{0}^{-1}+\hat{A}_{0}^{-1} \hat{A}^{\prime} \hat{\jmath} \widehat{G}^{-1},
$$

где $\widehat{G}_{0}^{-1}=-\hat{A}_{0}^{-1} \hat{g}^{-1}$ (здесь линия со стрелкой указывает матричную функцию, на которую действует дифференциальный оператор).

Уравнение (20) по своей структуре соответствует подобным уравнениям в методе производящего функционала для неприводимых частей функций Грина для моделей магнетиков или сильно коррелированных электронных систем [28]-[31]. Его решение можно искать, используя итерационную процедуру, в виде разложения, в котором слагаемые содержат разное число множителей $\hat{J}$.

Рассмотрим более детально первую итерацию. В этом случае

$$
\widehat{\Xi}^{-1}=\widehat{G}_{0}^{-1}+\hat{A}_{0}^{-1} \hat{\hat{A}^{\prime}} \hat{\hat{J}} \widehat{G} \widehat{G}_{0}^{-1}
$$

Полагая в конечном результате флуктуирующие поля равными нулю $\left(v_{\gamma} \rightarrow 0\right)$, для функции $\left(\widehat{\Xi}^{-1}\right)_{11}$ в частотном представлении получаем

$$
\left(\widehat{\Xi}^{-1}\right)_{11}\left(\omega_{n}\right)=\frac{1}{A_{0 B}}\left\{\left(g^{B 0}\right)^{-1}\left(\omega_{n}\right)\left[1-Q_{B}\left(\omega_{n}\right)-N_{B}\left(\omega_{n}\right)\right]+\frac{1}{A_{0 A}} \widetilde{S}_{B}\right\} .
$$

Здесь

$$
\begin{aligned}
Q_{B}\left(\omega_{n}\right) & =\left(\frac{\delta \Phi_{V}}{\delta v_{0 A}}\right)^{-2} \frac{1}{\beta} \sum_{\omega_{m}} \mathrm{e}^{i \omega_{m} 0^{+}} J_{B}\left(\omega_{m}\right) G_{\mathrm{c}}^{0 B}\left(\omega_{m}-\omega_{n}\right), \\
N_{B}\left(\omega_{n}\right) & =\frac{1}{\beta} \sum_{\omega_{m}} \mathrm{e}^{i \omega_{m} 0^{+}} J_{A}\left(\omega_{m}\right) G_{A}\left(\omega_{m}\right) G^{A B}\left(\omega_{m}-\omega_{n}\right) \frac{1}{D}, \\
\widetilde{S}_{B} & =\frac{1}{\beta} \sum_{\omega_{m}} \mathrm{e}^{i \omega_{m} 0^{+}} J_{A}\left(\omega_{m}\right) G_{A}\left(\omega_{m}\right),
\end{aligned}
$$

причем $D=\left.D(\tau)\right|_{v=0}$, где

$$
D(\tau)=\frac{\delta \Phi_{V}}{\delta v_{0 B}(\tau)} \frac{\delta \Phi_{V}}{\delta v_{0 A}(\tau)}-\frac{\delta \Phi_{V}}{\delta v(\tau)} \frac{\delta \Phi_{V}}{\delta \bar{v}(\tau)} .
$$

Бозонная функция Грина $G^{A B}$ магнонного типа и кумулянтная функция $G_{\mathrm{c}}^{0 B}$ суть вторые производные функционала $\Phi_{V}$ :

$$
\begin{gathered}
\frac{\delta^{2} \Phi_{V}}{\delta \bar{v}(\tau) \delta v\left(\tau_{2}\right)}=G^{A B}\left(\tau-\tau_{2}\right) \equiv\left\langle\mathcal{T}_{\tau} X^{A B}(\tau) X^{B A}\left(\tau_{2}\right)\right\rangle \\
\left.\frac{\delta^{2} \Phi_{V}}{\delta v_{0 B}(\tau) \delta v_{0 B}\left(\tau_{4}\right)}\right|_{v=0}=\left\langle\mathcal{T}_{\tau}\left(X^{00}+X^{B B}\right)_{\tau}\left(X^{00}+X^{B B}\right)_{\tau_{4}}\right\rangle-
\end{gathered}
$$




$$
-\left\langle\mathcal{T}_{\tau}\left(X^{00}+X^{B B}\right)_{\tau}\right\rangle\left\langle\mathcal{T}_{\tau}\left(X^{00}+X^{B B}\right)_{\tau_{4}}\right\rangle \equiv G_{\mathrm{c}}^{0 B}\left(\tau-\tau_{4}\right) .
$$

Выражение (22) для $\widehat{\Xi}^{-1}$, полученное в результате первой итерации, содержит формально слагаемые первого порядка по $J$. Вместе с тем в них входят полные бозонные функции $G^{A B}, G_{\mathrm{c}}^{0 B}$ и фермионная функция $\left.G_{A} \equiv G_{V}^{A 0,0 A}\right|_{v \rightarrow 0}$, которые должны определяться самосогласованно с использованием уравнений (3) и (4). Чтобы замкнуть систему уравнений, необходимо дополнить ее выражениями для бозонных функций $G^{A B}, G_{\mathrm{c}}^{0 B}$; эти выражения дают функциональную связь бозонных функций с фермионными функциями и когерентным потенциалом. На процедуре расчета бозонной функции $G^{A B}$ в используемом подходе мы остановимся ниже (раздел 5), а сначала рассмотрим упрощенную схему, которая приводит к предложенному ранее в [9], [32] GH3-приближению, являющемуся обобщением известного приближения Хаббард-III [35].

\section{GНЗ-ПРИБЛИЖЕНИЕ}

В этом разделе мы покажем, как перейти от первой итерации для $\widehat{\Xi}^{-1}$ в методе производящего функционала к GH3-приближению и к соответствующему выражению для одноузельной функции Грина $\left.G_{B} \equiv G_{V}^{B 0,0 B}\right|_{v \rightarrow 0}$, полученному при использовании метода уравнений движения и разновременных расцеплений [9], [32].

С этой целью возьмем в (22) фермионные и бозонные функции в нулевом приближении:

$$
\begin{gathered}
G^{A B}\left(\omega_{m}\right) \approx\left\langle X^{A A}-X^{B B}\right\rangle g^{A B}\left(\omega_{m}\right), \\
G_{\mathrm{c}}^{0 B}\left(\omega_{m}\right) \approx \beta \delta\left(\omega_{m}\right) A_{0 B}\left(1-A_{0 B}\right), \\
G_{A}\left(\omega_{m}\right) \approx A_{0 A} g^{A 0}\left(\omega_{m}\right), \quad G_{B}\left(\omega_{m}\right) \approx A_{0 B} g^{B 0}\left(\omega_{m}\right), \\
g^{p q}=\frac{1}{i \omega-\lambda_{p q}}, \quad \lambda_{p q}=\lambda_{p}-\lambda_{q},
\end{gathered}
$$

где $\lambda_{p}, \lambda_{q}$ - собственные значения невозмущенного одноузельного гамильтониана; $A_{0 \sigma}=\left\langle X^{00}+X^{\sigma \sigma}\right\rangle$. Путем несложных преобразований получим приближенное выражение для $(\widehat{\Xi})_{11}^{-1}$, а затем в соответствии с (3) и одноузельную функцию Грина $G_{B}[26]$ :

$$
G_{B}\left(\omega_{n}\right)=\frac{A_{0 B}}{\left(g^{B 0}\right)^{-1}\left(\omega_{n}\right)-\frac{P_{B}\left(\omega_{n}\right)}{A_{0 B}}+\frac{\widetilde{S}_{B}}{A_{0 B}}-J_{B}\left(\omega_{n}\right)} .
$$

Здесь

$$
P_{B}\left(\omega_{n}\right)=\frac{1}{\beta} \sum_{\omega_{m}} \mathrm{e}^{i \omega_{m} 0^{+}} J_{A}\left(\omega_{m}\right) \frac{\left\langle X^{A A}-X^{B B}\right\rangle}{i \omega_{m}-i \omega_{n}-\lambda_{A B}}
$$

- функция, которая в диаграммном изображении соответствует петле, содержащей линии когерентного потенциала $J_{A}$ и невозмущенной бозонной функции $g^{A B}$, идущие в противоположных направлениях:

$$
P_{B}\left(\omega_{n}\right)=\left\langle X^{A A}-X^{B B}\right\rangle \times-\cdots
$$


Здесь линия со стрелкой - когерентный потенциал $J_{A}$, линия с треугольником невозмущенная функция $g^{A B}$.

Записывая когерентный потенциал $J_{\sigma}$ в представлении Лемана,

$$
J_{\sigma}\left(\omega_{n}\right)=-\frac{1}{\pi} \lim _{\eta \rightarrow 0^{+}} \int_{-\infty}^{+\infty} \frac{\operatorname{Im} J_{\sigma}\left(\omega^{\prime}+i \eta\right) d \omega^{\prime}}{i \omega_{n}-\omega^{\prime}},
$$

и суммируя по частоте $\omega_{m}$, получим

$$
\begin{aligned}
& P_{B}\left(\omega_{n}\right)=\frac{\left\langle X^{A A}+X^{B B}\right\rangle}{2} J_{A}\left(i \omega_{n}+\lambda_{A B}\right)+\frac{\left\langle X^{A A}-X^{B B}\right\rangle}{2} Y_{B}\left(\omega_{n}\right), \\
& Y_{B}\left(\omega_{n}\right)=\frac{1}{2 \pi} \int_{-\infty}^{+\infty} d \omega^{\prime} \frac{\left[-2 \operatorname{Im} J_{A}\left(\omega^{\prime}+i 0^{+}\right)\right]}{i \omega_{n}-\omega^{\prime}+\lambda_{A B}} \operatorname{th} \frac{\beta \omega^{\prime}}{2} .
\end{aligned}
$$

Формула (32), в которую подставлены формулы (36), совпадает с выражением для функции $G_{B}$, полученным в GH3-приближении в работе [9] (имеется соответствие $P_{B}\left(\omega_{n}\right) \leftrightarrow-R_{B}(\omega) ; \widetilde{S}_{B} \leftrightarrow-V_{A} \varphi_{B}$ в принятых там обозначениях). Если пренебречь функцией $P_{B}$, выражение (32) сводится к приближению модифицированного сплава (см. работу [36]).

\section{5. БОЗОННАЯ ФУНКЦИЯ ГРИНА}

Процедура расчета обратной неприводимой части $\widehat{\Xi}^{-1}$ согласно формуле $(22)$ включает определение бозонной функции Грина $G^{A B}\left(\omega_{n}\right)$. При переходе к GH3-приближению используется невозмущенная функция $G_{0}^{A B}\left(\omega_{n}\right)$. Более строгий подход может базироваться на получении этой функции в виде функционала, зависящего от когерентного потенциала и фермионной функции Грина, с последующим самосогласованным ее расчетом. В методе производящего функционала можно использовать для этой цели способ, предложенный в [31], в основе которого лежит дифференцирование по полям средних от соответствующих бозонных операторов Хаббарда.

Для среднего $\left\langle\mathcal{T}_{\tau} X^{A B}(\tau)\right\rangle$ используем рекуррентную форму (типа (15)) теоремы Вика. Действуя с левой стороны обратной функцией $\left(g^{A B}\right)^{-1}\left(\tau^{\prime \prime}-\tau\right)$, дифференцируя по полю $v\left(\tau^{\prime}\right)$ и полагая после этого фиктивные поля равными нулю, придем к уравнению

$$
\begin{aligned}
\int_{0}^{\beta} d \tau\left(g^{A B}\right)^{-1}\left(\tau^{\prime \prime}-\tau\right)\left\langle\mathcal{T}_{\tau} X^{A B}(\tau) X^{B A}\left(\tau^{\prime}\right)\right\rangle=\delta\left(\tau^{\prime \prime}-\tau^{\prime}\right)\left\langle\mathcal{T}_{\tau}\left(X^{A A}-X^{B B}\right)_{\tau^{\prime \prime}}\right\rangle+ \\
\quad+\left.\int_{0}^{\beta} d \tau_{2} J_{B}\left(\tau_{2}-\tau^{\prime \prime}\right) \frac{\delta}{v\left(\tau^{\prime}\right)}\left\langle\mathcal{T}_{\tau} X^{A 0}\left(\tau^{\prime \prime}\right) X^{0 B}\left(\tau_{2}\right)\right\rangle\right|_{V \rightarrow 0}- \\
\quad-\left.\int_{0}^{\beta} d \tau_{2} J_{A}\left(\tau^{\prime \prime}-\tau_{2}\right) \frac{\delta}{v\left(\tau^{\prime}\right)}\left\langle\mathcal{T}_{\tau} X^{A 0}\left(\tau_{2}\right) X^{0 B}\left(\tau^{\prime \prime}\right)\right\rangle\right|_{V \rightarrow 0}
\end{aligned}
$$

С другой стороны, уравнение для этой же функции $\left\langle\mathcal{T}_{\tau} X^{A B}(\tau) X^{B A}\left(\tau^{\prime}\right)\right\rangle$ можно получить, дифференцируя среднее от оператора $\left\langle\mathcal{I}_{\tau} X^{B A}\left(\tau^{\prime}\right)\right\rangle$ по полю $\bar{v}(\tau)$. В этом случае будем иметь

$$
\int_{0}^{\beta} d \tau^{\prime}\left\langle\mathcal{T}_{\tau} X^{A B}(\tau) X^{B A}\left(\tau^{\prime}\right)\right\rangle\left(g^{A B}\right)^{-1}\left(\tau^{\prime}-\tau^{\prime \prime}\right)=\delta\left(\tau^{\prime \prime}-\tau\right)\left\langle\mathcal{T}_{\tau}\left(X^{A A}-X^{B B}\right)_{\tau^{\prime \prime}}\right\rangle-
$$




$$
\begin{aligned}
& -\left.\int_{0}^{\beta} d \tau_{2} J_{A}\left(\tau_{2}-\tau^{\prime \prime}\right) \frac{\delta}{\bar{v}(\tau)}\left\langle\mathcal{T}_{\tau} X^{B 0}\left(\tau^{\prime \prime}\right) X^{0 A}\left(\tau_{2}\right)\right\rangle\right|_{V \rightarrow 0}+ \\
& +\left.\int_{0}^{\beta} d \tau_{2} J_{B}\left(\tau^{\prime \prime}-\tau_{2}\right) \frac{\delta}{\bar{v}(\tau)}\left\langle\mathcal{T}_{\tau} X^{B 0}\left(\tau_{2}\right) X^{0 A}\left(\tau^{\prime \prime}\right)\right\rangle\right|_{V \rightarrow 0}
\end{aligned}
$$

Решения уравнений (37) и (38) совпадают, если вариационные производные по фиктивным полям найдены точно. Если используются аппроксимации, результат в общем случае зависит от выбора под знаком среднего исходного недиагонального оператора.

Перенесем операцию дифференцирования в (37) или (38) на обратную фермионную функцию Грина $\widehat{G}^{-1}$ (как это сделано в [28]-[30], см. также [31]) и воспользуемся тем, что в нулевом по $\hat{J}$ приближении

$$
\frac{\delta}{\delta v}\left(\widehat{\Xi}^{-1}\right)_{21}=-\left[\hat{A}_{12}^{\prime}\left(\hat{A}_{0}^{-1}\right)_{21}\right]\left(\hat{g}^{-1}\right)_{11}-\left(\hat{A}_{0}^{-1}\right)_{22}\left[\hat{A}_{12}^{\prime}\left(\hat{g}^{-1}\right)_{21}\right] .
$$

В результате получим

$$
\begin{aligned}
\int_{0}^{\beta} d \tau_{2} & J_{A}\left(\tau^{\prime \prime}-\tau_{2}\right) \frac{\delta}{v\left(\tau^{\prime}\right)}\left\langle\mathcal{T}_{\tau} X^{A 0}\left(\tau_{2}\right) X^{0 B}\left(\tau^{\prime \prime}\right)\right\rangle \approx \\
\approx & \int_{0}^{\beta} \frac{d \tau_{2} d \tau_{3} d \tau_{4}}{D} J_{A}\left(\tau^{\prime \prime}-\tau_{2}\right) G^{A 0}\left(\tau_{2}-\tau_{3}\right) G^{A B}\left(\tau_{3}-\tau^{\prime}\right) \times \\
& \times\left(g^{B 0}\right)^{-1}\left(\tau_{3}-\tau_{4}\right) G^{B 0}\left(\tau_{4}-\tau^{\prime \prime}\right)- \\
& -\int_{0}^{\beta} d \tau_{2} d \tau_{3} d \tau_{4} J_{A}\left(\tau^{\prime \prime}-\tau_{2}\right) G^{A 0}\left(\tau_{2}-\tau_{3}\right) \delta\left(\tau_{3}-\tau^{\prime}\right) \delta\left(\tau_{3}-\tau_{4}\right) \times \\
& \times G^{B 0}\left(\tau_{4}-\tau^{\prime \prime}\right) \frac{\left\langle X^{00}+X^{B B}\right\rangle}{D}, \quad G^{\sigma 0} \equiv G_{\sigma} .
\end{aligned}
$$

Используя данный прием в уравнениях (37) и (38) и переходя к частотному представлению, мы можем легко найти их решения и представить искомую функцию Грина в виде

$$
G^{A B}\left(\omega_{n}\right)=\frac{\left\langle X^{A A}-X^{B B}\right\rangle-R\left(\omega_{n}\right)}{\left(g^{A B}\right)^{-1}-M\left(\omega_{n}\right)},
$$

где функция $R\left(\omega_{n}\right)$ в числителе имеет смысл концевой части, а $M\left(\omega_{n}\right)$ - массовый оператор. Явный вид этих функций зависит от приближения и выбора исходного оператора при записи уравнений. Ниже мы приводим полученные результаты для случая $J_{B}=0$ (ФК-предел), рассматривая оба варианта; при этом используется диаграммное представление.

1. Уравнение (37); исходное среднее $\left\langle\mathcal{T}_{\tau} X^{A B}\right\rangle$ :

$$
R^{(1)}\left(\omega_{n}\right)=\frac{1}{A_{0 A}} \times\left[-C_{B 0-}\right],
$$




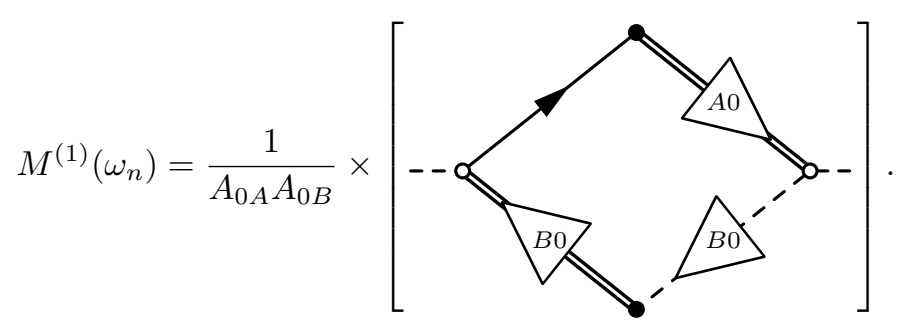

2. Уравнение (38); исходное среднее $\left\langle\mathcal{T}_{\tau} X^{B A}\right\rangle$ :

$$
M^{(2)}\left(\omega_{n}\right)=\frac{1}{A_{0 A} A_{0 B}} \times\left[\omega_{n}\right)=\frac{1}{A_{0 B}} \times[
$$

На приведенных диаграммах двойные линии соответствуют полным функциям Грина (их конкретный вид указан в треугольнике); пунктирные линии обозначают обратную невозмущенную функцию $g^{-1}$.

Два набора функций $R^{(1)}, M^{(1)}$ и $R^{(2)}, M^{(2)}$ формируют несколько разные приближения. Аналогично [31] можно также использовать следующие симметризированные выражения для $R\left(\omega_{n}\right)$ и $M\left(\omega_{n}\right)$ :

$$
M\left(\omega_{n}\right)=\frac{M^{(1)}\left(\omega_{n}\right)+M^{(2)}\left(\omega_{n}\right)}{2}, \quad R\left(\omega_{n}\right)=\frac{R^{(1)}\left(\omega_{n}\right)+R^{(2)}\left(\omega_{n}\right)}{2} .
$$

\section{6. СПЕКТРАЛЬНЫЕ ПЛОТНОСТИ$$
\rho_{B} \text { И } \rho_{A B} \text { В ФК-ПРЕДЕЛЕ }\left(t_{B}=0\right)
$$

Мы рассчитываем спектральные функции, выражающиеся через мнимые части соответствующих функций Грина, для фермионной и бозонной ветвей спектра численно на основании полученных выше уравнений. Расчет выполняется на действительных непрерывных частотах, для этого используется аналитическое продолжение $i \omega_{n} \rightarrow \omega+i 0^{+}$.

Чтобы вычислить сумму

$$
I\left(\omega_{n}\right)=\frac{1}{\beta} \sum_{\omega_{m}} J_{A}\left(\omega_{m}\right) G^{A 0}\left(\omega_{m}\right) G^{B 0}\left(\omega_{m}-\omega_{n}\right),
$$

которая соответствует петле функций Грина на диаграмме (42), применим аналогично (35) представление Лемана. Разлагая затем на простые дроби и суммируя по 
нечетным (фермиевским) частотам, после замены $i \omega_{n} \rightarrow \omega+i 0^{+}$получим

$$
\begin{aligned}
I\left(\omega+i 0^{+}\right)=- & \frac{1}{\pi} \int_{-\infty}^{+\infty} d \omega^{\prime \prime} \frac{\operatorname{Im} A\left(\omega+\omega^{\prime \prime}+i 0^{+}\right)}{\mathrm{e}^{\beta\left(\omega+\omega^{\prime \prime}\right)}+1} G^{B 0}\left(\omega^{\prime \prime}-i 0^{+}\right)- \\
& -\frac{1}{\pi} \int_{-\infty}^{+\infty} d \omega^{\prime \prime} \frac{\operatorname{Im} G^{B 0}\left(\omega^{\prime \prime}+i 0^{+}\right)}{\mathrm{e}^{\beta \omega^{\prime \prime}}+1} A\left(\omega+\omega^{\prime \prime}+i 0^{+}\right),
\end{aligned}
$$

где $A\left(\omega_{m}\right)=J_{A}\left(\omega_{m}\right) G^{A 0}\left(\omega_{m}\right)$. Другие суммы по частотам в аналитических выражениях для диаграмм (43) и (45) преобразуются в интегралы по частотам с помощью аналогичной процедуры. При этом, чтобы ее использовать в случае (43), необходимо разбить произведение $\left(g^{B 0}\right)^{-1}\left(\omega_{m}\right) G^{B 0}\left(\omega_{m}\right)$ на слагаемые: $\left(g^{B 0}\right)^{-1} G^{B 0}=$ $\left\langle X^{00}+X^{B B}\right\rangle-\widetilde{M}_{B} G^{B 0}$, введя согласно уравнению Дайсона массовый оператор $\widetilde{M}_{B}$ для функции Грина $G^{B 0}$ (явное выражение для $\widetilde{M}_{B}$ можно получить из формулы (32)).

Для расчета фермионной функции Грина используется выражение (32), которое возникает в методе разновременных расщеплений. Однако в этом случае бозонные функции Грина, которые в [26] были взяты в нулевом приближении (34), рассчитываются на основе уравнений, полученных в методе производящего функционала. Поэтому выражение для функции $P_{B}\left(\omega_{n}\right)$ записывается в следующем виде:

$$
P_{B}\left(\omega_{n}\right)=\frac{1}{\beta} \sum_{\omega_{m}} \mathrm{e}^{i \omega_{m} 0^{+}} J_{A}\left(\omega_{m}\right) G^{A B}\left(\omega_{m}-\omega_{n}\right) .
$$

Данная функция представляется в виде интеграла по частоте аналогично (48) (с учетом бозе-распределения при суммировании по четным частотам):

$$
\begin{aligned}
P_{B}\left(\omega+i 0^{+}\right)=- & \frac{1}{\pi} \int_{-\infty}^{+\infty} d \omega^{\prime \prime} \frac{\operatorname{Im} J\left(\omega+\omega^{\prime \prime}+i 0^{+}\right)}{\mathrm{e}^{\beta\left(\omega+\omega^{\prime \prime}\right)}+1} G^{A B}\left(\omega^{\prime \prime}-i 0^{+}\right)+ \\
& +\frac{1}{\pi} \int_{-\infty}^{+\infty} d \omega^{\prime \prime} \frac{\operatorname{Im} G^{A B}\left(\omega^{\prime \prime}+i 0^{+}\right)}{\mathrm{e}^{\beta \omega^{\prime \prime}}-1} J_{A}\left(\omega+\omega^{\prime \prime}+i 0^{+}\right) .
\end{aligned}
$$

В результате полная система уравнений, которая решается самосогласованно, формируется уравнением для $G^{A B}(\omega)(41)$, где функции $R(\omega), M(\omega)$ определяются с помощью процедуры, аналогичной (48), уравнениями для $G_{A}(\omega), G_{B}(\omega)(32)$ (при этом для $G_{A}(\omega)$ производится замена индексов $\left.A \leftrightarrow B\right)$, соотношением (50) и выражением для $\widetilde{S}_{B}$,

$$
\widetilde{S}_{B}=-\frac{1}{\pi} \int_{-\infty}^{+\infty} \frac{d \omega}{\mathrm{e}^{\beta \omega}+1} \operatorname{Im}\left[J_{A}\left(\omega+i 0^{+}\right) G_{A}\left(\omega+i 0^{+}\right)\right] .
$$

Ниже при численных расчетах мы ограничиваемся случаем $J_{B}=0(\Phi \mathrm{K}$-предел); функция $G_{A}(\omega)$ определяется при этом простым выражением

$$
G_{A}(\omega)=\frac{A_{0 A}}{\omega+\mu_{A}-J_{A}(\omega)},
$$

являющимся точным [3], [9]. Систему дополняет уравнение, вытекающее из уравнений (3) и (4), которое дает общую связь одноузельной функции $G_{A}$ с когерентным 
потенциалом $J_{A}$; вид этой связи зависит от типа кристаллической решетки. В случае решетки Бете с полуэллиптической плотностью состояний

$$
\rho_{\sigma}(\varepsilon)=\frac{2}{\pi W_{\sigma}^{2}} \sqrt{W_{\sigma}^{2}-\varepsilon^{2}}
$$

где $W_{\sigma}$ - полуширина невозмущенной зоны, имеется простая связь $J_{\sigma}(\omega)=W_{\sigma}^{2} G_{\sigma} / 4$ (в нашем случае $\left.W_{B}=0\right)$. Окончательно система уравнений замыкается термодинамическими соотношениями, которые определяют концентрации частиц и дают их связь с химическими потенциалами:

$$
n_{\sigma}=\frac{1}{\pi} \int_{-\infty}^{+\infty} d \omega \frac{-\operatorname{Im} G_{\sigma}\left(\omega+i 0^{+}\right)}{\mathrm{e}^{\beta \omega}+1} .
$$

Заметим, что в случае ФК-модели зависимости химических потенциалов от концентраций можно рассчитать, не используя соотношений (54), поскольку известно точное выражение для термодинамического потенциала [37]-[39]. На основании этого факта было выполнено детальное исследование термодинамики модели в различных термодинамических режимах [32], [40], [41] (см. также [42], где рассмотрение проводилось с использованием псевдоспинового формализма). Известно, что ФК-модель может описывать фазовые переходы, связанные с фазовым расслоением, а также с появлением модулированных фаз при конечных значениях $U$ [3], [41]. При $U \rightarrow \infty$ модулированные фазы исчезают, и остаются только переходы между однородными фазами. В частности, точные термодинамические соотношения на языке двухсортного решеточного газа были получены в пределе $U \rightarrow \infty$ в работе [32].

С другой стороны, имеются также точные методы расчета спектральных функций $\rho_{B}$ локализованных частиц для ФК-модели [3], [43]. Вместе с тем из-за их громоздкости и вычислительных трудностей рассматривается в основном лишь симметричный случай половинного заполнения [43], [44]. Мы используем развитую в настоящей работе приближенную схему для исследования плотности состояний $\rho_{B}$ в более широком диапазоне параметров, а точные термодинамические соотношения привлекаются для установления области применимости подхода.

Ранее в [9] путем сравнений с точными результатами для ФК-модели было установлено, что GH3-приближение дает хорошее описание термодинамики модели (зависимостей химических потенциалов от концентраций) при высоких концентрациях локализованных частиц $n_{B}$ и отрицательных значениях $\mu_{A} \lesssim 0$ химического потенциала подвижных частиц (что соответствует их низким концентрациям $n_{A}$ ). Это приближение давало также лучшие результаты при повышении температуры. В настоящей работе численные расчеты охватывают диапазон температур, включающий области термодинамической неустойчивости (фазовые переходы первого рода, расслоение фаз), а также температуры выше критических. В работе [32] путем анализа точных термодинамических соотношений для $\Phi \mathrm{K}$-модели при $U \rightarrow \infty$ было установлено, что упомянутые фазовые переходы возможны при $T<T_{\mathrm{c}} \approx 0.0601 W_{A}$. Исходя из этих данных мы провели численный расчет фермионных и бозонных спектральных функций

$$
\rho_{B}(\omega)=-\frac{1}{\pi} \operatorname{Im} G_{B}\left(\omega+i 0^{+}\right),
$$




$$
\rho_{A B}(\omega)=\frac{1}{\pi} \operatorname{Im} G^{A B}\left(\omega+i 0^{+}\right),
$$

при трех температурах: $T=0.02 W_{A}, T=0.06 W_{A}$ и $T=0.20 W_{A}$. Были рассмотрены также три варианта нахождения бозонной функции Грина: 1) на основе функций $R^{(1)}, M^{(1)}$, см. формулы $\left.(42),(43) ; 2\right)$ на основе $R^{(2)}, M^{(2)}$, см. формулы (44), (45); 3) на основе их симметризованных выражений, см. формулу (46).
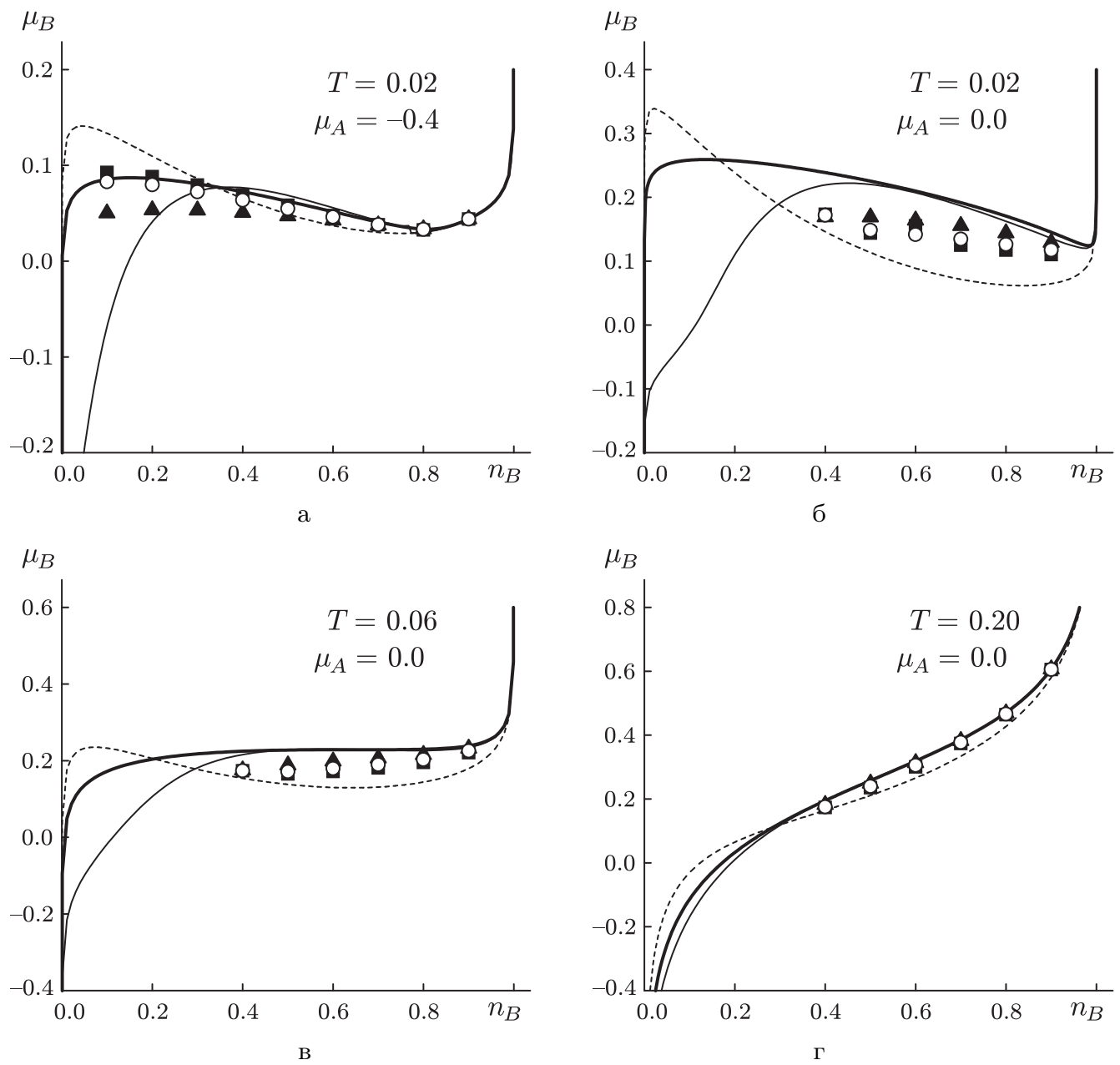

Рис. 1. Химический потенциал $\mu_{B}$ как функция концентрации $n_{B}$ в приближении модифицированного сплава - штриховая линия, GH3-приближении [9] - тонкая сплошная линия и в самосогласованном GH3-приближении: вариант 1 - $\mathbf{\square}$, вариант 2 - $\mathbf{\Delta}$, симметризованный вариант - о; для сравнения приведен точный результат [32] - жирная сплошная линия. Параметры расчета: $\rho_{\sigma}^{0}(t)$ для решетки Бете, $W_{A}=1, W_{B}=0$.

7 Теоретическая и математическая физика, т. 154, № 1, 2008 г. 
На рис. 1 приведены зависимости $\mu_{B}\left(n_{B}\right)$, рассчитанные с помощью соотношений (54), в сравнении с точными результатами, полученными ранее термодинамическим путем [32], а также с результатами расчета $\mu_{B}\left(n_{B}\right)$ в приближении модифицированного сплава и GH3-приближении [9]. Установлено, что при $\mu_{A}=-0.4$ наш самосогласованный расчет даже при низких температурах $(T=0.02)$ улучшает GH3-приближение, и найденная зависимость $\mu_{B}\left(n_{B}\right)$ находится в хорошем соответствии с точной кривой, воспроизводя области метастабильных состояний и неустойчивости и описывая тем самым фазовый переход с достаточной точностью (заметим, что участки кривых с $\partial \mu_{B} / \partial n_{B}<0$ на графиках при $T=0.02$ соответствуют неустойчивости, связанной с упомянутым выше фазовым переходом первого рода, при котором изменяются скачком концентрации частиц). При $\mu_{A}=0$ (что соответствует повышению концентрации подвижных частиц $n_{A}$ по сравнению со случаем $\left.\mu_{A}=-0.4\right)$ остается проблема описания системы при низких концентрациях $n_{B}$. В этом случае мы имеем удовлетворительные результаты лишь при высоких температурах $\left(\mu_{A}=0, T=0.2\right)$.

Кривые на рис. 2а иллюстрируют различные приближения для бозонных плотностей состояний, которые соответствуют первому или второму варианту выбора приоритетов при построении уравнений для функции $G^{A B}$, а также симметризованному варианту. Вид кривых в разных приближениях отличается; общим, однако, является то, что все они дают пик в окрестности $\omega+\mu_{A}-\mu_{B}=0$. Имеется также довольно широкое фоновое размытие, соразмерное с полушириной $W_{A}$ невозмущенной плотности зонных состояний. Следует также отметить, что в отличие от фермионной плотности состояний функция $\rho_{A B}(\omega)$ может менять знак.

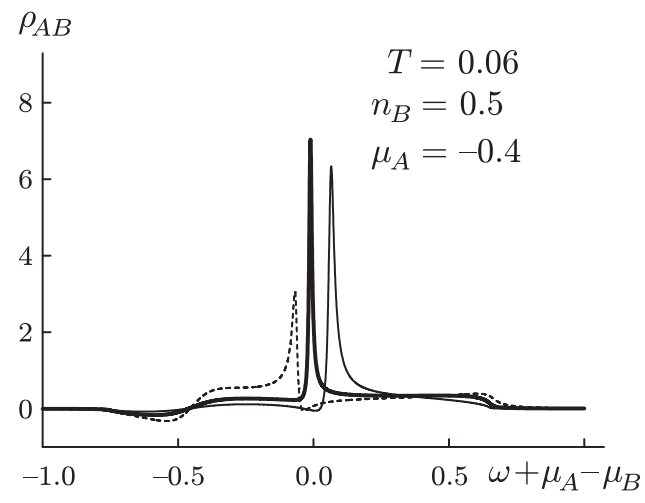

a

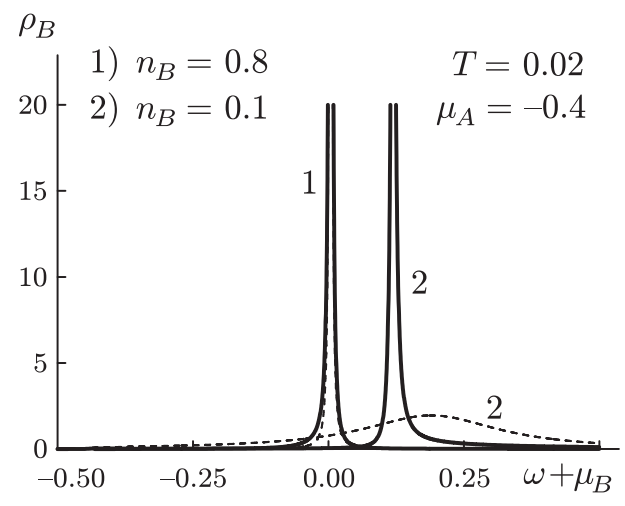

б

Рис. 2. Бозонная плотность $\rho_{A B}(\omega)$ в разных приближениях: вариант 1 тонкая сплошная линия, вариант 2 - штриховая линия, симметризованный вариант - жирная сплошная линия (а). Фермионная спектральная функция $\rho_{B}(\omega)$ в GH3-приближении (штриховые линии) и в симметризованном варианте самосогласованного GH3-приближения (сплошные линии) при разных концентрациях $n_{B}$ (б). 


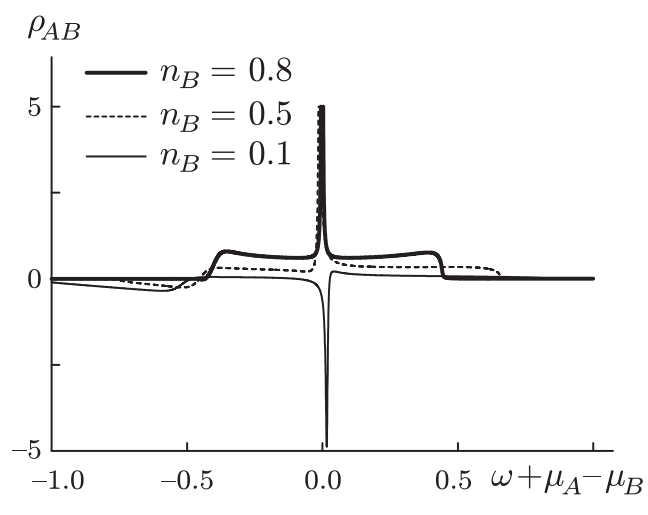

a

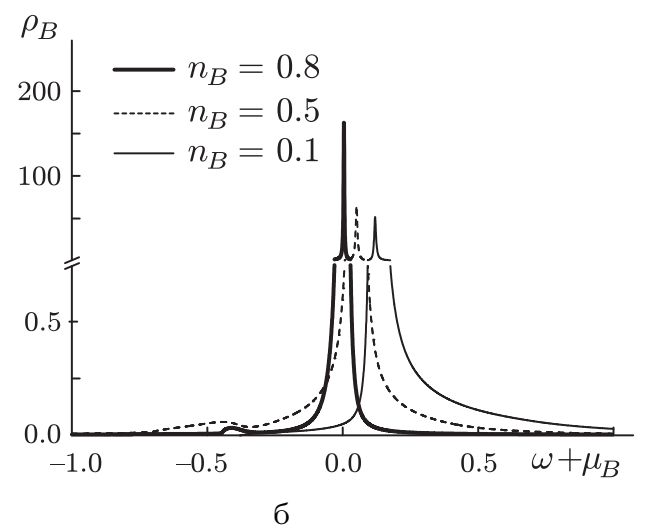

Рис. 3. Спектральные функции $\rho_{A B}(\omega)$ и $\rho_{B}(\omega)$ при различных концентрациях $n_{B}$ локализованных частиц (симметризованный вариант самосогласованного GH3-приближения); параметры расчета: $T=0.02, \mu_{A}=-0.4$.
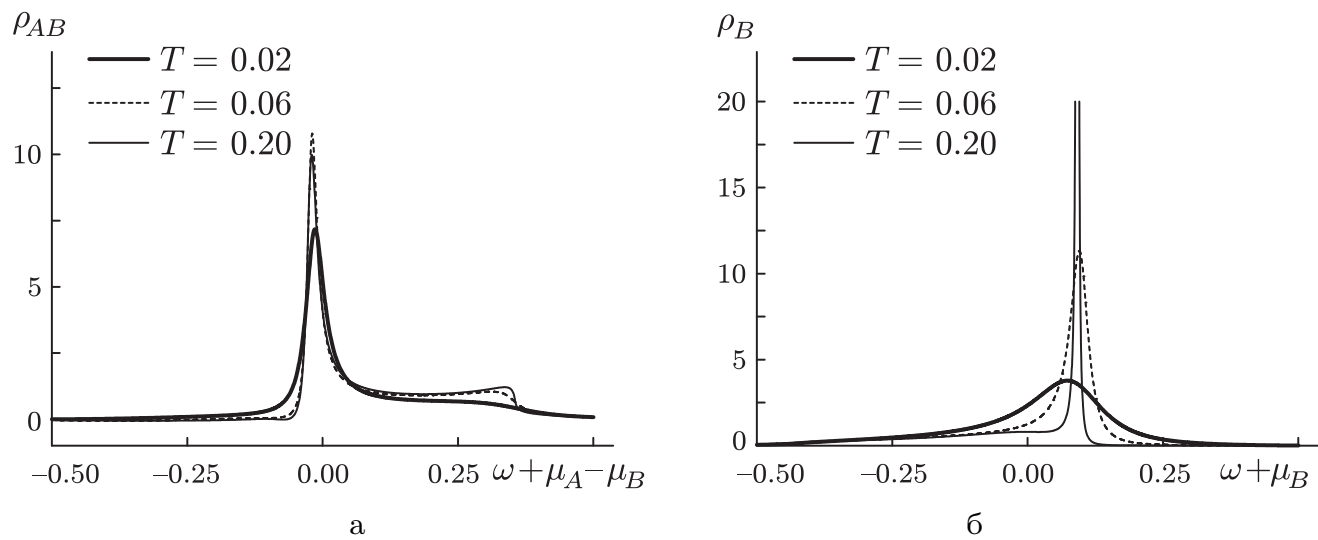

Рис. 4. Спектральные функции $\rho_{A B}(\omega)$ и $\rho_{B}(\omega)$ при различных температурах (симметризованный вариант самосогласованного GH3-приближения); параметры расчета: $n_{B}=0.8, \mu_{A}=0$.

Наилучшие термодинамические результаты дает вариант с симметризованными уравнениями (46), так как нельзя выделить, какой тип диаграмм $\left(M^{(1)}\right.$ или $\left.M^{(2)}\right)$ является более важным; поэтому далее все результаты расчетов приводятся для симметризованного случая. На рис. $2 б$ приведены графики для фермионной плотности $\rho_{B}(\omega)$ (плотности состояний неподвижных частиц), полученные путем самосогласованного расчета на основании системы уравнений (32), (41). Для сравнения показан также вид кривых $\rho_{B}(\omega)$ в обычном GH3-приближении. Видно, что при концентрации $n_{B}=0.8$, когда обычное GH3-приближение дает хорошие термодинамические 
результаты, кривые почти совпадают. С другой стороны, при $n_{B}=0.1$ применение GH3-приближения приводит для $\mu_{B}\left(n_{B}\right)$ к сильно отличающемуся от точного результату: спектр $\rho_{B}(\omega)$ получается сильно размытым. При этом самосогласованная схема (которую мы можем называть самосогласованным GH3-приближением), улучшая существенно ход кривой $\mu_{B}\left(n_{B}\right)$ в этой области, дает одновременно узкий профиль плотности состояний $\rho_{B}$.

На основе анализа рассчитанных зависимостей $\mu_{B}\left(n_{B}\right)$ (рис. 1) можно выделить область значений параметров, при которых модифицированное GH3-приближение приводит к удовлетворительным результатам (высокие температуры $T \gtrsim T_{\text {c }}$, не слишком низкие концентрации $n_{B}$, невысокие концентрации $\left.n_{A}\left(\mu_{A} \lesssim 0\right)\right)$. Выделив такие случаи, мы рассчитали поведение бозонной $\rho_{A B}$ и фермионной $\rho_{B}$ плотностей состояний при различных концентрациях $n_{B}$ (рис. 3) или температурах (рис. 4). Из рис. 3 видно, что при возрастании $n_{B}$ до значения 0.8 (что соответствует низкой концентрации подвижных частиц $\left.n_{A} \sim 0.005\right)$ бозонный спектр становится почти симметричным, а фермионный приближается по форме к невозмущенному спектру (дельта-пику). Однако даже в этом случае имеется, кроме резкого пика, довольно широкое фоновое размытие бозонной и фермионной функций. При уменьшении концентрации $n_{B}$ (соответственно, увеличении $n_{A}$ до $n_{A} \sim 0.22$ при $n_{B}=0.1$ ) размытие становится больше, и спектры приобретают асимметричный вид.

Существенная асимметрия спектральных функций видна и на рис. 4, где приведены графики $\rho_{A B}(\omega)$ и $\rho_{B}(\omega)$ для случая $\mu_{A}=0$ при различных температурах. Имеет место заметное уширение пиков и усиление фонового размытия при повышении температуры. Отметим, что приближенные плотности состояний удовлетворяют правилам сумм, а именно интеграл по частоте (площадь под кривой) равен $1-n_{A}$ для фермионной функции $\rho_{B}(\omega)$ и равен $n_{B}-n_{A}$ для бозонной функции $\rho_{A B}(\omega)$. В отличие от фермионных плотностей состояний, знак бозонных плотностей может изменяться. В частности, при $n_{B}<n_{A}$ функция $\rho_{A B}(\omega)$ в основном отрицательна (см. рис. 3 , случай $n_{B}=0.1$ ); при этом в нулевом приближении, когда $\left(G^{A B}\right)^{0}=\left\langle X^{A A}-X^{B B}\right\rangle g^{A B}\left(\omega_{n}\right)$, функция $\rho_{A B}(\omega) \leqslant 0$ во всем интервале частот.

Как уже было отмечено выше, точные результаты для фермионных плотностей состояний частиц ФК-модели известны только в некоторых случаях (большинство результатов относится к симметричному случаю половинного заполнения). Это обусловлено тем, что при использовании точной схемы расчета возникают вычислительные проблемы при увеличении потенциала отталкивания $U$ или при понижении температуры [44]. Поэтому мы можем сравнивать различные приближения с точными данными, лишь приближаясь к половинному заполнению. Такое сравнение в случае GH3-приближения было сделано в [10] для АMХ-модели в ФК-пределе при конечных значениях $U$ и $n_{A}=n_{B}=0.5, \mu_{A}=\mu_{B}=U / 2$. Было достигнуто лучшее согласие с точными данными работы [43] при достаточно высоких температурах и больших потенциалах $U$. Когда $U \rightarrow \infty$ и химические потенциалы конечны по модулю, верхняя хаббардовская подзона удаляется на бесконечность и остается лишь одна нижняя подзона; она и является предметом рассмотрения в настоящей работе. 


\section{7. ЗАКЛЮЧЕНИЕ}

Сформулированный в работе подход к решению одноузельной задачи в методе динамического среднего поля направлен на развитие приближенной аналитической схемы расчета функций Грина электронных моделей с хаббардовскими взаимодействиями. Использование метода производящего функционала Каданова-Бейма позволяет получить систематическую итерационную процедуру определения неприводимых частей фермионных и бозонных одноузельных функций Грина путем формального разложения по степеням когерентного потенциала. При этом сами функции находятся из системы интегральных уравнений. Уже первая итерация дает результаты, которые являются обобщением полученных ранее в рамках метода уравнений движения (в картине вспомогательных фермионов) при использовании разновременного расцепления функций высшего порядка [9], [32].

Нулевое приближение по бозонной функции Грина, которая входит в неприводимую часть для фермионной функции, соответствует GH3-приближению. Последнее было детально исследовано для AMX в работах [9], [10], [32]. В ФК-пределе (когда $\left.W_{B} \rightarrow 0\right)$ мы сравнили наши результаты с результатами точных расчетов [9] при половинном заполнении. Как отмечалось выше, GH3-приближение оказалось удовлетворительным при высоких $T$, а также при низких концентрациях $n_{A}$ подвижных частиц и достаточно высоких концентрациях $n_{B}$ локализованных частиц.

Самосогласованное GH3-приближение основано на более полном учете вклада бозонных возбуждений (при $U \rightarrow \infty$ имеется лишь ветвь, описываемая спектральной плотностью $\rho_{A B}$ ); массовый оператор для бозонной функции Грина имеет однопетлевую структуру. Такой подход позволяет расширить область применения схемы GH3-приближения. Остается тем не менее проблема описания спектра при низких $T$ и в областях концентраций, недоступных данному приближению. Возможный путь улучшения теории может состоять, в частности, в переходе к высшим приближениям при расчете бозонной функции Грина, например, в духе обобщенного приближения хаотических фаз путем суммирования последовательностей петлевых вкладов в массовый оператор (в диаграммном представлении это может приблизить к структуре, получающейся при точном расчете одноузельной зарядовой восприимчивости в методе динамического среднего поля [45]).

Недостатком GH3-приближения является также отсутствие в спектральных плотностях особенностей типа пика Кондо [10]. Попытка исправить эту ситуацию путем сложных одновременных расцеплений функций Грина была сделана Ешке и Котляром [21] в рамках метода уравнений движения. Как нами показано в [26], такой подход также можно рассматривать как обобщение GH3-подхода, но иного рода: в этом случае эффективно учитываются вклады более высокого порядка по когерентному потенциалу в неприводимые части фермионных функций Грина. Систематический анализ таких вкладов является на данный момент нерешенной задачей.

\section{Список литературы}

[1] Ю. А. Изюмов, УФН, 165:4 (1995), 403.

[2] A. Georges, G. Kotliar, W. Krauth, M. J. Rozenberg, Rev. Mod. Phys., 68 (1996), 13. 
[3] J. K. Freericks, V. Zlatić, Rev. Mod. Phys., 75 (2003), 1333.

[4] A. N. Kocharian, G. R. Reich, J. Appl. Phys., 76 (1994), 6127.

[5] D. Ueltschi, J. Stat. Phys., 116 (2004), 681.

[6] C. D. Batista, Phys. Rev. Lett., 89 (2002), 166403.

[7] G. Fáth, Z. Domański, R. Lemański, Phys. Rev. B, 52 (1995), 13910.

[8] C. A. Macedo, A. M. C. de Souza, Phys. Rev. B, 65 (2002), 153109.

[9] I. V. Stasyuk, O. B. Hera, Phys. Rev. B, 72 (2005), 045134.

[10] I. V. Stasyuk, O. B. Hera, Eur. Phys. J. B, 48 (2005), 339.

[11] W. Metzner, D. Vollhardt, Phys. Rev. Lett., 62 (1989), 324.

[12] M. Jarrell, Phys. Rev. Lett., 69 (1992), 168.

[13] M. J. Rozenberg, X. Y. Zhang, G. Kotliar, Phys. Rev. Lett., 69 (1992), 1236.

[14] A. Georges, W. Krauth, Phys. Rev. Lett., 69 (1992), 1240.

[15] M. Caffarel, W. Krauth, Phys. Rev. Lett., 72 (1994), 1545.

[16] Q. Si, M. J. Rozenberg, G. Kotliar, A. E. Ruckenstein, Phys. Rev. Lett., 72 (1994), 2761.

[17] R. Bulla, Adv. Solid State Phys., 40 (2000), 169.

[18] K. Held, I. A. Nekrasov, G. Keller et al., Psi-k Newsletter, 56 (2003), 65.

[19] A. Georges, AIP Conf. Proc., 715 (2004), 3.

[20] I. V. Stasyuk, Condens. Matter Phys., 3:2(22) (2000), 437.

[21] H. O. Jeschke, G. Kotliar, Phys. Rev. B, 71 (2005), 085103.

[22] X. Dai, K. Haule, G. Kotliar, Phys. Rev. B, 72 (2005), 045111.

[23] A. M. Shvaika, Phys. Rev. B, 62 (2000), 2358.

[24] N. E. Bickers, D. L. Cox, J. W. Wilkins, Phys. Rev. B, 36 (1987), 2036.

[25] M. B. Zölf, T. Pruschke, J. Keller et al., Phys. Rev. B, 61 (2000), 12810.

[26] I. V. Stasyuk, O. B. Hera, Condens. Matter Phys., 9:3(47) (2006), 587.

[27] L. P. Kadanoff, G. Baym, Quantum Statistical Mechanics, Benjamin, New York, 1962.

[28] Y. A. Izyumov, N. I. Chaschin, Phys. Metals and Metallography, 92 (2001), 451.

[29] Y. A. Izyumov, N. I. Chaschin, Phys. Metals and Metallography, 92 (2001), 531.

[30] Y. A. Izyumov, N. I. Chaschin, V. Y. Yushankhai, Phys. Rev. B, 65 (2002), 214425.

[31] Y. A. Izyumov, N. I. Chaschin, D. S. Alexeev, F. Mancini, Eur. Phys. J. B, 45 (2005), 69.

[32] I. V. Stasyuk, O. B. Hera, Condens. Matter Phys., 6:1(33) (2003), 127.

[33] I. V. Stasyuk, A. M. Shvaika, Ukr. J. Phys., 47 (2002), 975.

[34] Д. Н. Зубарев, УФН, 71 (1960), 71.

[35] J. Hubbard, Proc. Roy. Soc. Ser. A, 281 (1964), 401.

[36] M. Potthoff, T. Herrmann, T. Wegner, W. Nolting, Physica Status Solidi (b), 210 (1998), 199.

[37] U. Brandt, C. Mielsch, Z. Phys. B, 75 (1989), 365.

[38] U. Brandt, C. Mielsch, Z. Phys. B, 79 (1990), 295.

[39] U. Brandt, C. Mielsch, Z. Phys. B, 82 (1991), 37.

[40] J. K. Freericks, C. Gruber, N. Macris, Phys. Rev. B, 60 (1999), 1617.

[41] B. M. Letfulov, Eur. Phys. J. B, 11 (1999), 423.

[42] I. V. Stasyuk, A. M. Shvaika, J. Phys. Stud., 3 (1999), 177.

[43] U. Brandt, M. P. Urbanek, Z. Phys. B, 89 (1992), 297.

[44] J. K. Freericks, V. M. Turkowski, V. Zlatić, Phys. Rev. B, 71 (2005), 115111.

[45] A. M. Shvaika, Phys. C, 341-348 (2000), 177. 